\title{
Correlation between HGF/c-Met and Notch1 signaling pathways in human gastric cancer cells
}

\author{
KUO-HUNG HUANG ${ }^{1,2}$, I-CHENG SUNG ${ }^{4}$, WEN-LIANG FANG ${ }^{1,3}$, CHIN-WEN CHI $^{4,6}$, \\ TIEN-SHUN YEH ${ }^{5}$, HSIN-CHEN LEE ${ }^{4}$, PEN-HUI YIN ${ }^{6}$, ANNA FEN-YAU LI ${ }^{8}$, \\ CHEW-WUN WU ${ }^{1,3}$, YI-MING SHYR ${ }^{1,3}$ and MUH-HWA YANG ${ }^{2,7}$ \\ ${ }^{1}$ Division of General Surgery, Department of Surgery, Taipei Veterans General Hospital, Taipei 112; \\ ${ }^{2}$ Institute of Clinical Medicine, School of Medicine, National Yang-Ming University; ${ }^{3}$ School of Medicine, \\ National Yang-Ming University, Taipei 112; ${ }^{4}$ Department and Institute of Pharmacology, and \\ ${ }^{5}$ Department of Anatomy and Cell Biology, School of Medicine, National Yang-Ming University, \\ Taipei 112; ${ }^{6}$ Department of Medical Research, ${ }^{7}$ Division of Medical Oncology, Department of Oncology, \\ and ${ }^{8}$ Department of Pathology, Taipei Veterans General Hospital, Taipei 112, Taiwan, R.O.C.
}

Received November 30, 2017; Accepted May 15, 2018

DOI: $10.3892 /$ or.2018.6447

\begin{abstract}
In recent decades, research concerning gastric carcinogenesis has rapidly progressed. It is evident that hepatocyte growth factor (HGF) is clinically related to gastric cancer progression and metastasis. In addition, previous studies have found that expression of Notch ligand Jagged1 is correlated with the poor prognosis of gastric cancer. However, the interaction between the HGF/c-Met and Notch1 signaling pathways remains unknown. In the present study, we found that gastric cancer patients with positive c-Met expression exhibited poorer overall survival than patients without c-Met expression $(\mathrm{P}=0.043)$ and that Jagged1 expression was significantly correlated with c-Met expression $(\mathrm{r}=0.301 ; \mathrm{P}=0.004)$ in human gastric cancer specimens. In addition, Jagged1 activity increased after HGF stimulation, which in turn increased the downstream expression of cyclooxygenase 2 (COX-2) in a timedependent manner. After knockdown of Notch1 intracellular domain (N1IC), HGF was found to increase the proliferation and migration ability in human gastric cancer cells. However, overexpression of N1IC still had no effect after HGF stimulation. Our study found a feedback loop between HGF/c-Met and Jagged1/Notch1 signaling. Furthermore, both HGF/c-Met and Notch1 signaling triggered COX-2 activity. These results suggest that gastric cancer progression is not associated with a unique signaling pathway and that a feedback loop may exist between the HGF/c-Met and Notch1 signaling pathways, which
\end{abstract}

Correspondence to: Dr Muh-Hwa Yang, Institute of Clinical Medicine, School of Medicine, National Yang-Ming University, No. 155, Sec. 2, Linong Street, Beitou, Taipei 112, Taiwan, R.O.C. E-mail: mhyang2@vghtpe.gov.tw

Key words: gastric cancer, hepatocyte growth factor, HGF/c-Met, Notch pathway, Jagged1 may result in therapeutic resistance. Therefore, multi-modality therapies should be considered for treating gastric cancer.

\section{Introduction}

Gastric cancer is a common malignancy of the gastrointestinal tract and has a high mortality rate worldwide. According to the World Health Organization, gastric cancer causes 74,000 deaths ( $~ 9.7 \%$ of total cancer mortality) per year worldwide. Although the incidence of gastric cancer has rapidly declined in recent decades, it is currently the third leading cause of cancer mortality in the world (GLOBOCAN 2012) (1). Surgical resection with radical lymph node dissection still plays an important role in gastric cancer therapy. Chemotherapy or targeted therapies are alternative therapeutic strategies for incurable gastric cancer or tumor recurrence after surgical resection. However, in the biological molecular field, gastric carcinogenesis remains poorly understood.

Hepatocyte growth factor (HGF), which is secreted by mesenchymal cells, is a potent mitogenic factor for hepatocytes. HGF binds to the c-Met receptor and activates tyrosine kinase signaling pathways. HGF targets and acts on epithelial cells and endothelial cells and is also associated with cell migration, proliferation and angiogenesis (2-4). Elevation of serum HGF levels and overexpression of the c-Met gene are often found in patients with more advanced tumors or metastasis $(5,6)$. The correlation between COX-2 and c-Met expression and human gastric cancer was found to be statistically significant. In addition, the overexpression of COX-2 and elevation of prostaglandin $E_{2}$ were found to be involved in the growth and metastasis of gastric cancer (2). These studies clearly show that HGF targets c-Met and regulates its biological function in gastric cancer.

Notch1 can act as an oncogene in breast, gastric, pancreatic and colon cancers, or as a tumor-suppressor gene in neuroendocrine tumors and skin cancer (7). Jagged1 is an intercellular ligand that activates the Notch1 signaling pathway (8). The 
activity of Notch ligand Jagged1 is associated with a poor prognosis in gastric cancer patients after surgery (3). Notch1 intracellular domain (N1IC), the intracellular activated form of the Notch1 receptor, translocates to the COX-2 promoter in the nucleus by binding $\mathrm{C}$ promoter binding factor 1 . Overexpression of N1IC was demonstrated to increase cell proliferation and migration (9). COX-2 expression promotes the production of prostaglandin $\mathrm{E}_{2}$. The interaction between intercellular Jagged1 and the Notch1 receptor induces COX-2 expression, which causes growth and metastasis in gastric cancer.

Notably, both the HGF/c-Met and Jagged1/Notch1 pathways are associated with COX-2 activity. COX-2 overexpression was found to be associated with tumor invasion depth (10), lymphovascular invasion, lymph node metastasis and more advanced gastric cancers (11). Elevated levels of COX-2 protein were found in gastric cancer tumors. Moreover, COX-2-specific inhibitor NS-398 and COX-2 knockdown suppressed the growth of human gastric cancer SC-M1 cells (12). However, the role of the interaction between the HGF/c-Met and Notch1 signaling pathway in gastric cancer cells remains obscure. The present study aimed to elucidate the influence of HGF on Jagged1/Notch signal transduction in SC-M1 gastric cancer cells.

\section{Materials and methods}

Surgical specimens. From January 2003 to December 2005, human gastric cancer samples were obtained from gastric cancer patients who underwent gastric surgery at the Department of Surgery, Taipei Veterans General Hospital. A total of 91 gastric cancer patients (including 59 males and 32 females, age from 33-84 years) were enrolled in this study and informed consent was obtained from all patients. Gastric tumors and adjacent gastric tissues were fixed overnight at $4^{\circ} \mathrm{C}$ with $4 \%$ neutral buffered paraformaldehyde, dehydrated, cleared with Histo-Clear II (National Diagnostics, Atlanta, GA, USA) and wax embedded. Five-micron sections were used for hematoxylin and eosin (H\&E) staining and immunohistochemical staining. This study was approved by the Institutional Review Board of Tapei Veterans General Hospital and informed consent was provided by all subjects.

Immunohistochemical staining of $c$-Met and Jaggedl. The localization of c-Met receptor and Jagged1 ligand in gastric tissues was detected using an avidin-biotin-peroxidase complex $(\mathrm{ABC})$ technique according to a protocol established and used for gastric cancer specimens $(2,3)$. Immunostaining of $\mathrm{HGF} / \mathrm{c}-\mathrm{Met}$ receptors in gastric cancer tissue was performed. Briefly, tissue sections were first microwaved in a sodium citrate buffer (10 mM, pH 6.0) and, were then treated with peroxidase blocking solution $\left(3 \% \mathrm{H}_{2} \mathrm{O}_{2}\right)$ for 5-10 min. Serum blocking solution (Histostain SP kit; Zymed Laboratories Inc., South San Francisco, CA, USA) was applied to remove endogenous peroxidase activity and to reduce nonspecific background staining. The antibodies used for tissue sections were as follows: polyclonal rabbit anti-c-Met (C-28) (1:200; cat. no. sc-161; Santa Cruz Biotechnology Inc., Dallas, TX, USA) at a 1:500 dilution and polyclonal rabbit anti-human Jagged1 antibody (cat. no. sc8303; Santa Cruz Biotechnology) at a 1:50 dilution. Slides were incubated overnight at $4^{\circ} \mathrm{C}$ in a moist chamber. The tissue sections were subsequently treated with post-primary block reagent: NovoLink polymer detection system (cat. no. RE7140-K; Leica Biosystems, Wetzlar, Germany) for $10 \mathrm{~min}$, streptavidin peroxidase-conjugated for $10 \mathrm{~min}, \mathrm{AEC}$ substrate chromogen for 5-10 min, and then counterstained with hematoxylin for $5 \mathrm{~min}$. Pre-immune rabbit IgG was used as a negative control. The distribution pattern of chromogen in tumor cells was examined by an experienced pathologist (A.F.-Y. Li).

The distribution of c-Met and Jagged1 proteins in human gastric cancer specimens was evaluated by a semi-quantitative system to calculate and estimate within the following arbitrary ranges: (-) no positive cells; (+) 1-25\%; (++) 26-75\%; and $(+++)>75 \%$.

Cell culture and transfection. Our previous study tested the Notch1 expression in SC-M1, AGS, NUGC3 and KATO III gastric cancer cell lines. The expression level of N1IC in SC-M1 gastric cancer cells was much more abundant than that in the other gastric cancer cell lines (3). Therefore, we chose SC-M1 cells for further experimentation. The overexpression of N1IC increased Notch activity in SC-M1 cells. Human gastric cancer SC-M1 cells were cultured in RPMI-1640 medium (Gibco; Thermo Fisher Scientific, Inc., Waltham, MA, USA) with $10 \%$ fetal bovine serum (FBS; HyClone Laboratory; GE Healthcare Life Sciences, Logan, UT, USA) and $0.1 \%$ gentamicin (Gibco; Thermo Fisher Scientific, Inc.). Our previous study showed that SC-M1 gastric cancer cell lines also express other Notch family members (Notch 1, 2, 3 and 4). N1IC expression was found to be significantly higher than the expression of other Notch family members $(13,14)$. Therefore, we choose N1IC for further experimentation. To establish stable expression of an HA-N1IC fusion protein in SC-M1 cells (SC-M1/HA-N1IC), a pcDNA-HA-N1IC expression plasmid was transfected into SC-M1 cells, and expression levels were screened by western blot analysis according to a previous established protocol (3). For the control, the pcDNA3 vector was also transfected into the SC-M1 cell line. The SC-M1/HA-N1IC and SC-M1/ pcDNA3 cell lines were selected with G418 $(400 \mu \mathrm{g} / \mathrm{ml}$; CalbioChem; EMD/Merck KGaA).

Drug treatment. Human gastric cancer SC-M1, SC-M1/ HA-N1IC and SC-M1/pcDNA3 cells were treated with HGF alone (0-25 ng/ml; Gibco; Thermo Fisher Scientific, Inc.), NS398 alone (50 $\mu \mathrm{M}$; Cayman Chemical Company, Ann Arbor, MI, USA), or HGF in combination with NS398. HGF promotes gastric cancer cell growth, and NS398 is a COX-2specific inhibitor that has anti-inflammatory and analgesic effects. To assess the effects on cell growth, the gastric cancer cell lines were treated with HGF and NS398 alone, or combined HGF and NS398 for 24, 48 or $72 \mathrm{~h}$. To assess the effects on migration, cells were treated with HGF $(25 \mathrm{ng} / \mathrm{ml})$ for $64 \mathrm{~h}$, and cell migration was observed at 8, 32 and $64 \mathrm{~h}$. For western blot analysis, the gastric cancer cell lines were incubated with HGF $(25 \mathrm{ng} / \mathrm{ml})$, and protein samples were collected at 1, 2, 4 and $24 \mathrm{~h}$ to analyze c-Met, Jagged 1 and COX-2 protein expression.

MTT assay. SC-M1 cells were seeded into a 96-well plate at a density of $1.2 \times 10^{4}$ cells/well and incubated for 
A

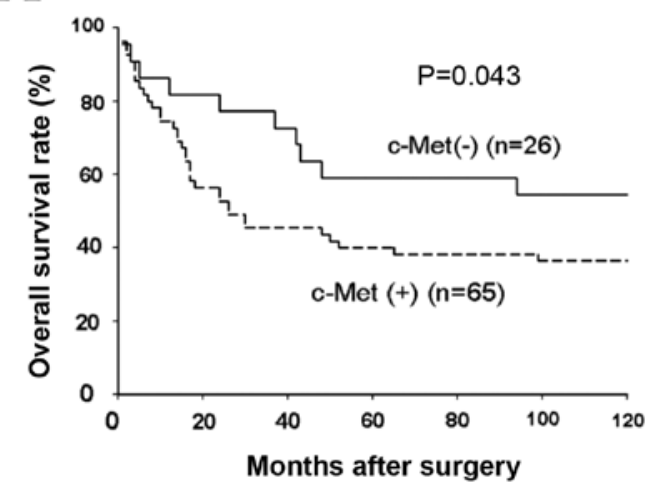

C

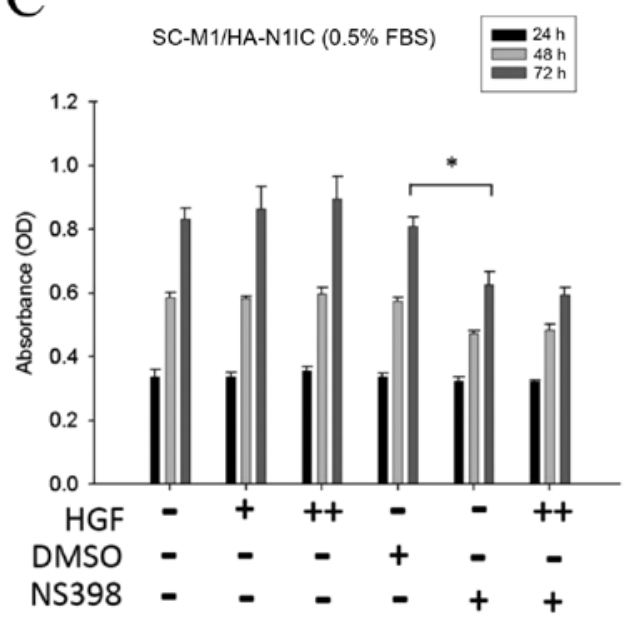

B
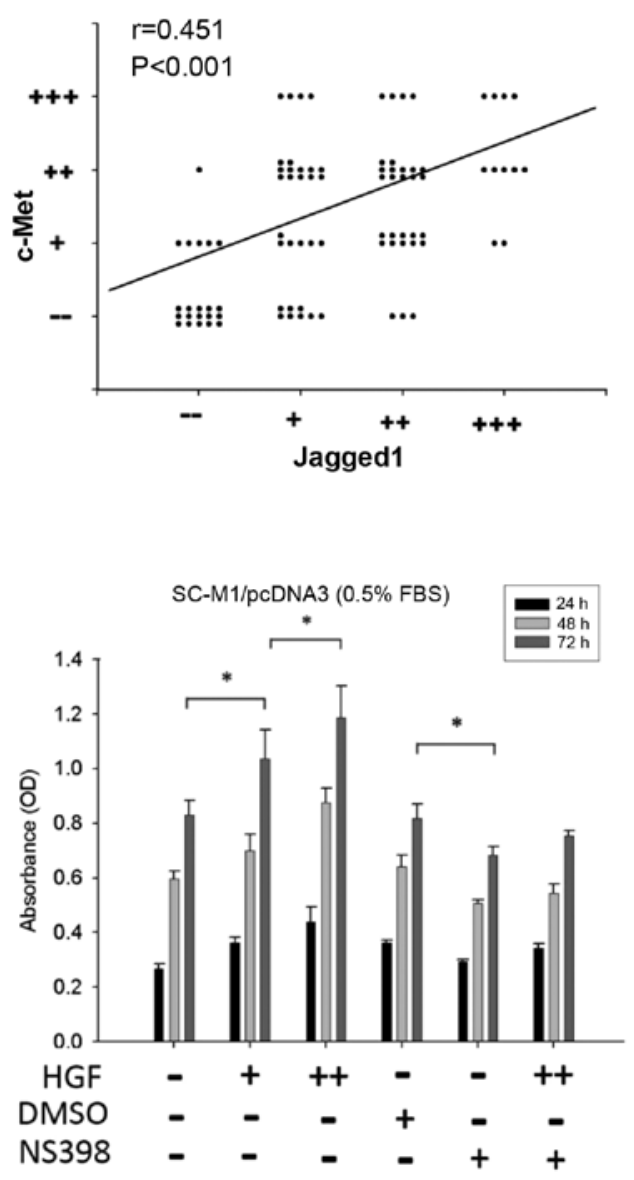

Figure 1. Clinical relevance of c-Met and Jagged1 expression in gastric cancer tissues. (A) Approximately 91 gastric cancer tissues were collected for immunohistochemical staining of c-Met and Jagged1. Patients with positive c-Met expression had poorer overall survival than patients without c-Met expression ( $\mathrm{P}=0.043)$. (B) Statistical correlation between c-Met and Jagged1 expression was compared using the linear regression model ( $\mathrm{P}=0.004)$. (C) Effect of HGF and NS398 on the growth of SC-M1/HA-N1IC and SC-M1/pcDNA3 cells. Transfected SC-M1 cells were cultured in the presence or absence of HGF (0, 5 or $25 \mathrm{ng} / \mathrm{dl})$ and NS398 $(0$ or $50 \mu \mathrm{M})$ for 3 days, and the cell growth was measured by MTT assay. Representative data are the average of 6 replicates. (HGF dosage: -, +, and ++ denotes 0,5 , and $25 \mathrm{ng} / \mathrm{dl}$, respectively; NS398 dosage: - and + denotes 0 and $50 \mu \mathrm{M}$, respectively; ${ }^{*} \mathrm{P}<0.01$ at $72 \mathrm{~h}$ ). HGF, hepatocyte growth factor; N1IC, Notch1 intracellular domain.

24 h. After treatment with HGF and/or NS398 for 24, 48, or $72 \mathrm{~h}$, the medium was replaced with $100 \mu \mathrm{l}$ of medium with 3-[4,5-dimethylthiazol-2-yl]-2,5-diphenyl-tetrazolium bromide (MTT). Then, the plate was incubated in a $37^{\circ} \mathrm{C}$ incubator. After discarding the medium, $100 \mu$ l dimethyl sulfoxide (DMSO) was added. The optical density was measured at an absorption wavelength of $570 \mathrm{~nm}$ by an ELISA reader.

Wound healing migration study. The migration ability of the drug- or vehicle-treated gastric cancer cells was evaluated by wound healing migration analysis. Culture-inserts (Ibidi-cells in focus, Munich, Germany) were placed in 6-well dishes, and $8 \times 10^{4}$ SC-M1 cells were seeded onto the inserts and incubated for $24 \mathrm{~h}$. Inserts were removed and gently washed to remove floating cells. After addition of $0.5 \%$ FBS medium with or without HGF $(25 \mathrm{ng} / \mathrm{ml})$, images of cell migration were captured at 8,32 and $64 \mathrm{~h}$. The percentage of the migration area was compared to the initial area after removing the insert. The migration area of gastric cancer cells was measured by ImageJ version 1.46 software (National Institutes of Health, Bethesda, MD, USA).
Transwell migration assay. Cell migration assays were analyzed using 24-well Transwell inserts with $5-\mu \mathrm{m}$ pores (Corning Costar, Cambridge, MA, USA). Approximately $5 \times 10^{4}$ cells were resuspended in $100 \mu 1$ of low serum (0.1\% FBS)-containing medium and loaded into the inserts with low serum-containing medium in the lower chamber. After incubating for $12 \mathrm{~h}$, either DMSO or HGF in low serum-containing medium at indicated concentrations was added to the inserts, which were then immediately shifted to new wells with high serum (20\% FBS)-containing medium to allow migration in a humidified $37^{\circ} \mathrm{C}$ incubator with $5 \%$ $\mathrm{CO}_{2}$ for $24 \mathrm{~h}$. SC-M1, SC-M1/HA-N1IC and SC-M1/pcDNA3 cells were then treated with HGF and/or NS398. After incubation for $24 \mathrm{~h}$, the inserts were removed, fixed in ice-cold methanol for $20 \mathrm{~min}$, and then stained using Liu staining solutions (Tonyar Biotech, Inc., Taoyuan, Taiwan). After staining, the cells that remained on the upper sides of the inserts were carefully wiped-off using rinsed cotton swabs. The migrated cells found on the bottom of the inserts were then imaged (at x100 magnification) and counted. Migrated cells from 10 random fields of each inserts were counted by 

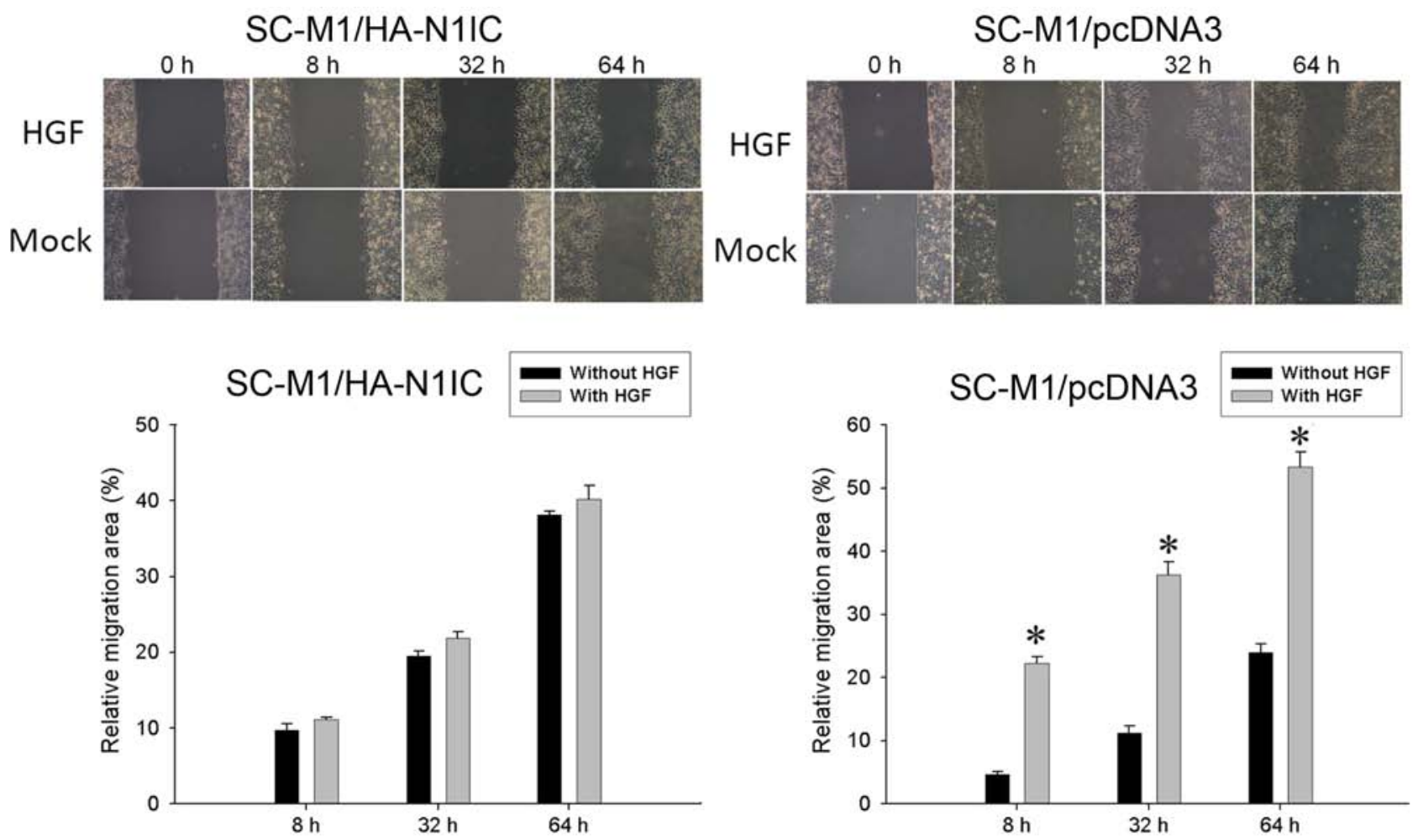

Figure 2. Wound healing migration of transfected SC-M1 cells was evaluated in the presence or absence of HGF (25 ng/d1). Relative migration area was estimated by the reduced square measure at 8,32 and $64 \mathrm{~h}$ compared to the square measure at $0 \mathrm{~h}$. ${ }^{*} \mathrm{P}<0.01$, HGF treatment compared to cells without HGF treatment. (The representative data are an average of 3 replicates). HGF, hepatocyte growth factor.

ImageJ version 1.46 software (National Institutes of Health) and normalized.

Western blot analysis. SC-M1 cells were treated with or without HGF (25 ng/ml) for 1,2 or $4 \mathrm{~h}$. The cells were mixed with NP-40 lysis buffer, and then added with mammalian protein extraction reagents (M-PER) in an ice bath. Cell lysates were collected after centrifugation at $23,220 \mathrm{x} \mathrm{g}$ for $10 \mathrm{~min}$ at $4^{\circ} \mathrm{C}$, and cell lysates were heated at $90^{\circ} \mathrm{C}$ for $5 \mathrm{~min}$. Protein concentrations were quantitatively determined using Bradford method. Protein $(30 \mu \mathrm{g})$ samples were electrophoresed on $6 \%$ (for c-Met and Jagged1) or 7.5\% (for COX-2) SDS-polyacrylamide gels. Each gel was then transferred to a polyvinylidene fluoride (PVDF) membrane (Immobilon-P; EMD Millipore, Bedford, MA, USA). The PVDF membrane was blocked with 5\% non-fat milk for $30 \mathrm{~min}$ and probed with rabbit anti-c-Met antibody (C-28) (cat. no. sc-161; Santa Cruz Biotechnology) at a 1:250 dilution, rabbit anti-Jagged1 antibody (cat. no. sc8303; Santa Cruz Biotechnology) at a 1:500 dilution, or mouse anti-COX-2 antibody (cat. no. 160112; Cayman Chemical Company) overnight at $4^{\circ} \mathrm{C}$. After washing with Tris-buffered saline containing $0.1 \%$ Tween-20 (TBS-T), the membranes were incubated with secondary anti-rabbit antibody (cat. no. 7074; Cell Signaling Technology, Danvers, MA, USA) at a 1:2,000 dilution. Enhanced protein bands were detected by a chemiluminescence kit (Amersham Life Science, Piscataway, NJ, USA).

Statistical analyses. All statistical analyses were carried out using SPSS software version 18.0 (SPSS, Inc., Chicago, IL, USA). Clinicopathological differences were compared with
Chi-square tests, and the correlation between Jagged1 and c-Met expression in each specimen was estimated with linear regression modeling. A P-value $<0.05$ was considered to indicate a statistically significant result.

\section{Results}

Expression of c-Met and Jaggedl expression in gastric cancer tissue in relation to survival. To evaluate the correlation between c-Met and Jagged1 in gastric cancer specimens, we collected samples from 91 gastric cancer patients in our study. The expression of c-Met and Jagged1 in gastric cancer tissue was analyzed by immunohistochemical staining of the 91 gastric cancer samples. While the positive c-Met expression rate was $71.4 \%$ (65/91), the positive Jagged1 expression rate was $76.9 \%$ (70/91). Patients with positive c-Met expression had poorer overall survival than patients with negative c-Met expression $(\mathrm{P}=0.043)$ (Fig. 1A). Our previous study showed that patients with positive Jagged1 expression also had poorer prognosis than patients with negative Jagged1 expression (3). Jagged1 expression was positively correlated with c-Met expression in the human gastric cancer tissues ( $\mathrm{P}=0.004)$ (Fig. 1B). Taken together, these results indicate a positive correlation between the c-Met and Jaggedl signaling pathways.

Effect of HGF stimulation on the growth of SC-M1/pcDNA3 and SC-MI/HA-NIIC cells. To evaluate the effect of HGF on the Notch1/Jagged1 pathway, N1IC-overexpressing (SC-M1/ HA-N1IC) and control (SC-M1/pcDNA3) human gastric cancer SC-M1 cells were used in our experiment. To reduce the influence of the growth factors in FBS, the concentration of FBS 
A

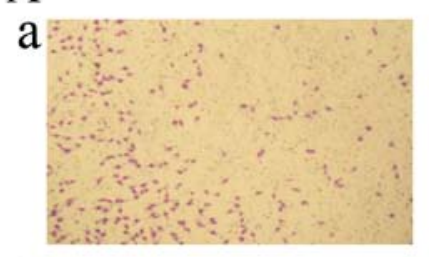

c

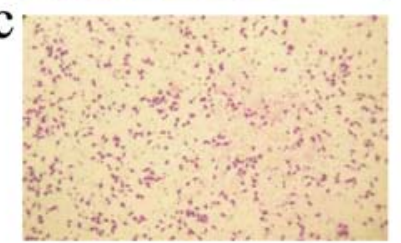

B

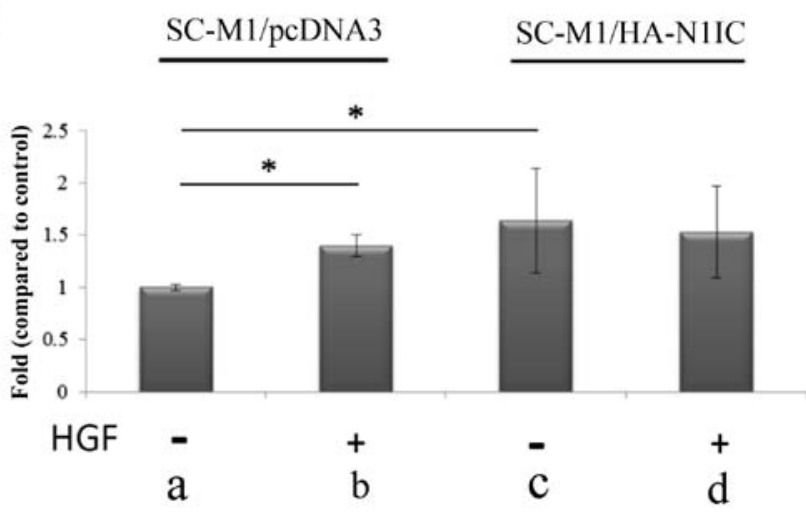

Figure 3. Migration analysis in SC-M1/HA-N1IC and SC-M1/pcDNA3 cells. (A) Transwell migration assay of SC-M1/pcDNA and SC-M1/HA-N1IC cells following treatment with/without HGF $(25 \mathrm{ng} / \mathrm{ml}$ ) for $24 \mathrm{~h}$. (a) SC-M1/ pcDNA3 cells, (b) SC-M1/pcDNA3 cells treated with HGF, (c) SC-M1/ HA-N1IC cells, (d) SC-M1/HA-N1IC cells treated with HGF. (B) Transwell migration assay of SC-M1/pcDNA3 and SC-M1/HA-N1IC cells following treatment with/without HGF $(25 \mathrm{ng} / \mathrm{ml})$ for $24 \mathrm{~h}$. Fold changes in the migration of gastric cancer cells from the respective groups. Cells were cultured in serum-free media for $24 \mathrm{~h}$ before Transwell assay. SC-M1/pcDN3A or SC-M1/HA-N1IC cells were treated with/without HGF for $24 \mathrm{~h}$, and then the migrated cells were counted. Total cell number was counted from 9 fields. ${ }^{*} \mathrm{P}<0.01$ compared to SC-M1/pcDNA3 cells. (Data are presented as the mean \pm SD of 2 replicates from three experiments). N1IC, Notch1 intracellular domain; HGF, hepatocyte growth factor.

was tapered to $0.5 \%$. Cells were treated with various concentration of HGF $(0,5$ or $25 \mathrm{ng} / \mathrm{ml})(-,+$ and ++ , respectively in the figures) and/or NS398 $(0$ or $50 \mu \mathrm{M})(-$ and + , respectively in the figures). The cell growth of SC-M1/pcDNA3 cells was increased in proportion to the elevation of HGF concentration. However, this phenomenon was not observed in the SC-M1/ HA-N1IC cells. The COX-2-specific inhibitor NS398 blocked cell growth in both SC-M1/HA-N1IC and SC-M1/pcDNA3 cells, and HGF-stimulated cell growth in SC-M1/pcDNA3 was also blocked by NS398 (Fig. 1C). These data showed that HGF can enhance the proliferation ability of gastric cancer with lower Notch1 (N1IC) activity. However, the effect of HGF treatment on proliferation in gastric cancer cells with constitutively overexpressed Notch1 (N1IC) was not obvious, such that the COX-2-specific inhibitor NS398 repressed the migration ability in gastric cancer cells with both lower and higher Notch1 (N1IC) activity.

Effect of HGF treatment on wound healing migration and Transwell migration assays in SC-M1/pcDNA3 and SC-M11
HA-NIIC cells. Next, we investigated the migration ability of gastric cancer cells using wound healing migration and Transwell migration assays. In the wound healing migration study, SC-M1/HA-N1IC and SC-M1/pcDNA3 cells were treated with HGF ( 0 or $25 \mathrm{ng} / \mathrm{ml}$ ). The relative migration area was increased after HGF treatment in SC-M1/pcDNA3 cells compared to HGF treatment in SC-M1/HA-N1IC cells (Fig. 2). Furthermore, we investigated the migration ability using a Transwell migration assay. Compared to the migration ability of SC-M1/pcDNA3 cells, SC-M1/HA-N1IC cell migration increased independently of HGF. The migration ability of SC-M1/pcDNA3 cells was increased after HGF treatment. However, there was no significant difference in the migration ability of SC-M1/HA-N1IC cells before and after HGF treatment (Fig. 3). NS398 treatment reduced the migration ability in the SC-M1/pcDNA3 and SC-M1/HA-N1IC cells (Fig. 4). These results suggest that HGF can enhance the migration ability of gastric cancers with lower Notch1 (N1IC) activity. However, the effect of HGF on gastric cancer cells with constitutively overexpressed Notch1 (N1IC) was not obvious. The COX-2-specific inhibitor NS398 can repress the migration ability in gastric cancer cells with both low and overexpressed N1IC.

Effect of HGF treatment on the expression of Jagged 1 and COX-2 proteins in Notch1-overexpressing, control and wildtype (WT) SC-MI cells. First, we compared the expression of c-Met and, Jagged1 by western blot analysis in Notch1overexpressing, control and WT SC-M1 gastric cancer cells. Jagged1 expression in SC-M1/WT and SC-M1/pcDNA3 cells was lower than that in SC-M1/HA-N1IC cells, but c-Met protein expression was lower in SC-M1/pcDNA3 and SC-M1/ HA-N1IC cells than that in the SC-M1/WT cells. The expression of c-Met protein in the SC-M1/pcDNA3 cells was higher than that in the SC-M1/HA-N1IC cells. Reduced expression of c-Met protein was observed in Notch1-overexpressing SC-M1 cells (Fig. 5A). Second, we treated gastric cancer cells with HGF and examined the effects over time. After HGF $(25 \mathrm{ng} / \mathrm{ml})$ treatment for 1,2 or $4 \mathrm{~h}$, cell lysates were collected for western blot analysis. HGF stimulated the expression of Jagged 1 and COX-2 proteins in the SC-M1/pcDNA3 cells in a time-dependent manner. However, Jagged1 and COX-2 proteins levels were not obviously increased in the SC-M1/ HA-N1IC cells. A time-dependent increase of Jagged1 was observed in the HGF-stimulated SC-M1 cells but not in the Notch1-overexpressing SC-M1 cells (Fig. 5B). COX-2 expression was elevated in the SC-M1/WT and SC-M1/pcDNA3 cells after HGF treatment. However, no significant change in COX-2 expression was observed in the SC-M1/HA-N1IC cells (Fig. 5C). Taken together, these results imply that a feedback loop exists between the HGF/c-Met and Notch1/Jagged1 signaling pathways.

\section{Discussion}

Our results showed that patients with positive c-Met expression had poorer overall survival than patients without positive expression. Jagged1 expression was correlated with HGF/c-Met expression, and HGF increased the expression of Jagged1, which in turn enhanced the Notch1 signaling 
A
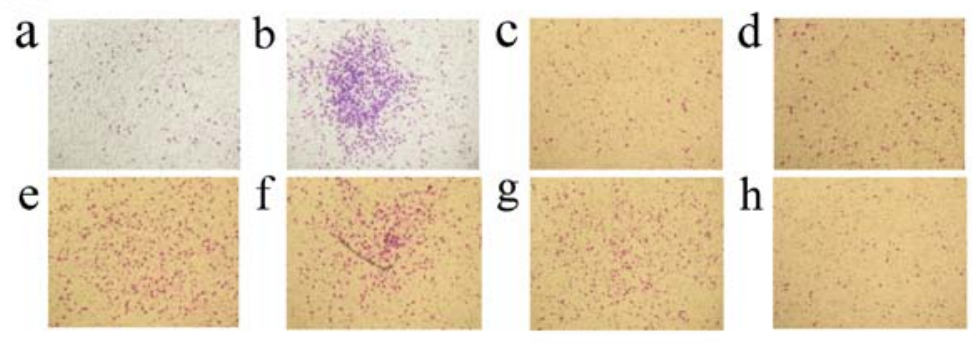

B

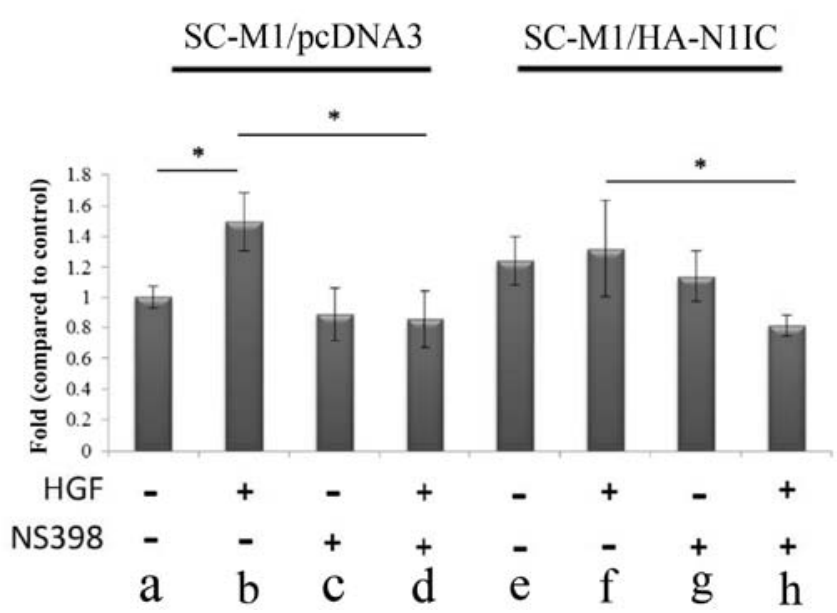

Figure 4. Migration analysis in SC-M1/HA-N1IC and SC-M1/pcDNA3 cells. (A) The migration of transfected SC-M1 cells was evaluated in the presence or absence of HGF $(25 \mathrm{ng} / \mathrm{ml})$, and/or NS398 $(50 \mu \mathrm{M})$ in SC-M1/pcDNA and SC-M1/HA-N1IC cells for $24 \mathrm{~h}$. The migration ability of (a) SC-M1/pcDNA3 (b) SC-M1/pcDNA + HGF (c) SC-M1/pcDNA3 + NS398 (d) SC-M1/pcDNA3 + HGF and NS398. (e) SC-M1/HA-N1IC (f) SC-M1/HA-N1IC + HGF (g) SC-M1/ HA-N1IC + NS-398 (h) SC-M1/HA-N1IC + HGF and NS398. (B) Migration of transfected SC-M1 cells was evaluated following treatment with HGF (0 and $25 \mathrm{ng} / \mathrm{ml})$, with/without NS-398 $(50 \mu \mathrm{M})$ in SC-M1/pcDNA3 and SC-M1/HA-N1IC cells for $24 \mathrm{~h}$. Migration ability of (a) SC-M1/pcDNA3 cells, (b) SC-M1/ pcDNA3 + HGF, (c) SC-M1/pcDNA3 + NS398, (d) SC-M1/pcDNA3 + HGF and NS398; (e) SC-M1/HA-N1IC cells, (f) SC-M1/HA-N1IC + HGF, (g) SC-M1/ HA-N1IC + NS398, (h) SC-M1/HA-N1IC + HGF and NS398. Fold changes in migration of gastric cancer cells from respective groups. Cells were cultured in serum-free media for $24 \mathrm{~h}$ before the Transwell assay. SC-M1/pcDNA3 or SC-M1/HA-N1IC cells were treated with/without HGF in the presence/absence of COX-2 inhibitor NS398 for $24 \mathrm{~h}$, and then the migrated cells were counted. Total cell number were counted from nine fields. "P<0.01 compared to SC-M1/ pcDNAs. (Data are presented as the mean \pm SD of 2 replicates from 3 experiments). N1IC, Notch1 intracellular domain; HGF, hepatocyte growth factor.

pathway, resulting in the expression of COX-2 and increased proliferation and migration ability in gastric cancer cells. However, the overexpression of N1IC suppressed the expression of c-Met and the sensitivity of HGF. The HGF/c-Met and Jagged1/Notch1 signaling pathways have the same target that triggers COX-2 mRNA expression to further manufacture the key product of COX-2 proteins.

Our previous studies have supported the concept of the parallel HGF/cMET/COX-2 (2) and Notch1/Jagged1/COX-2 (3) pathways. Both the gain and loss of c-MET and Notch were found to regulate $\mathrm{COX}-2$ expression, and the overexpression of N1IC or Jagged1 extracellular domain in SC-M1 cells had the same effect $(3,9)$. HGF induced Jagged1 expression, which increased in a time-dependent manner. Therefore, HGF could increase Jagged1 ligand activity through the HGF/c-Met signaling pathway. Our results support findings of a previous study by Stella et al, who reported that constitutive activation of Notch can inhibit HGF-dependent Ras activation in a model using Drosophila (15). Furthermore, activation of c-Met stimulates Notch signaling by inducing Notch ligand. Hence, an alternative loop exists in which HGF/c-Met induces the activation of Notch signaling through Jagged1 ligand, whereas Notch overexpression represses the expression of c-Met.
HGF plays an important role in the regulation of growth and metastasis of tumor cells. Our previous study showed that gastric cancer patients with high serum HGF had poorer prognosis than those with low serum HGF $(16,17)$. In addition, HGF was found to bind to the c-Met receptor and activates the tyrosine kinase signaling pathway, resulting in cell invasion and metastasis. COX-2 inhibitor NS398 was found to repress the proliferation and migration ability in human gastric cancer SC-M1 cells and inhibit the expression of COX-2 protein, which is stimulated by HGF (18). Uen et al (19) reported that patients with elevated c-Met mRNA expression in peripheral blood had poorer prognosis than patients with negative c-Met expression. Overexpression of c-Met increased the sensitization of gastric cancer cells to HGF, which in turn resulted in cell invasion and metastasis (20). In addition, Yamamoto et al (21) reported that $\mathrm{COX}-2$ protein expression was significantly elevated in human gastric cancer and associated with lymphatic invasion and metastasis. Thus, it is conceivable that HGF/c-Met has a transcriptional effect on the COX-2 promotor to induce the end product COX-2 protein to modulate the behavior of gastric cancer cells.

The Jagged1/Notch1 signaling pathway also plays an important functional role in regulating tumor cell proliferation and 
A

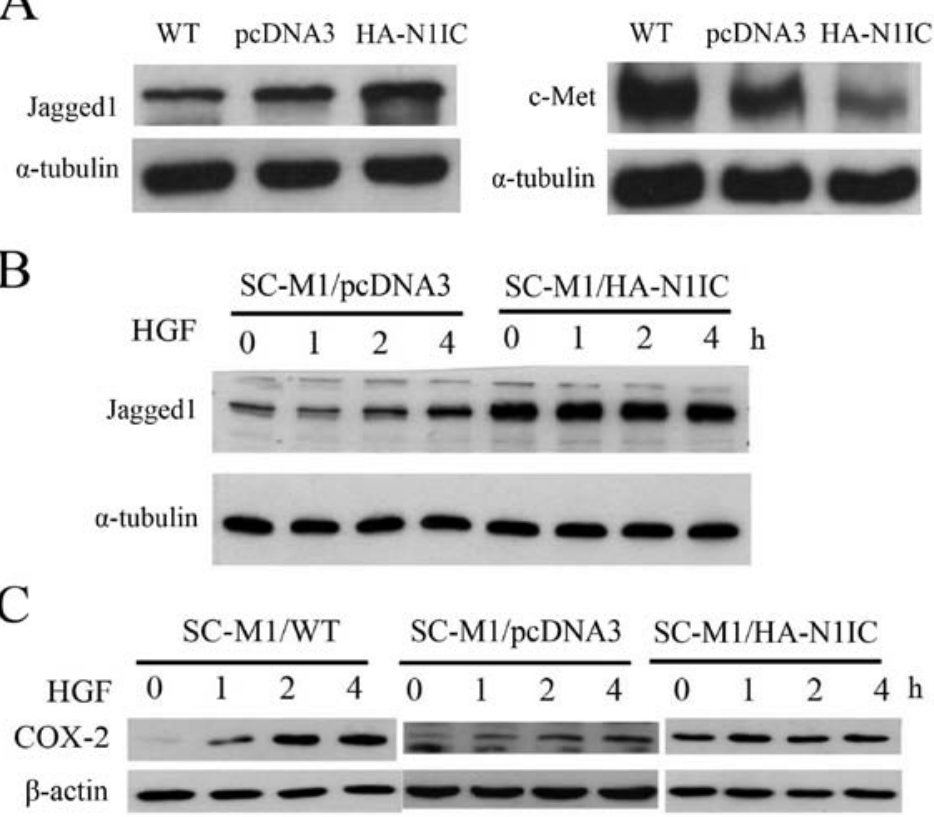

Figure 5. Western blot analysis of the protein expression of c-Met, Jagged1 and COX-2 in SC-M1 cells. After HGF (25 ng/dl) treatment at the indicated times $(1,2$ or $4 \mathrm{~h}$ ), the cells were collected, lysed and analyzed for c-Met, Jagged1 and COX-2 expression. (A) Jagged1 and c-Met expression in SC-M1/wild-type (WT), SC-M1/pcDNA3 and SC-M1/HA-N1IC cells. Jagged1 expression in SC-M1/WT and SC-M1/pcDNA3 cells was lower than that in SC-M1/HA-N1IC cells. The expression of c-Met protein was relatively low in SC-M1/HA-N1IC cells. (B) HGF/c-Met signaling induced Jagged1 expression in SC-M1/pcDNA3 cells in a time-dependent manner. (C) HGF/c-Met signaling induced COX-2 expression of SC-M1/pcDNA3 cells in a time-dependent sequence. HGF, hepatocyte growth factor; N1IC, Notch1 intracellular domain.

migration. Previous studies have revealed that Notch ligand Jagged1 and c-Met expression both positively correlate with COX-2 expression (23). We found a positive correlation between c-Met and Jagged1 in human gastric cancer tissues. In addition to their regulation of COX-2 protein, there is a circuit loop through which HGF increases Jagged1 expression, which in turn activates Notch1 activity. Therefore, elucidating the mechanism involved in the downstream regulation of c-Met and the interplay of Notch and c-Met signaling could help to understand the transcription effect in gastric cancer.

HGF regulates cellular signaling pathways through its interaction with c-Met. HGF was shown to elicit prolonged phosphorylation of growth factor receptor-bound protein 2 (GRB2)-associated-binding protein 1 (GAB1) and to lead to prolonged activation of mitogen-activated protein kinases (MAPK) $(22,23)$. Notch signaling, triggered by the MAPK pathway, was reported to play an important role in tumor angiogenesis $(24,25)$. Jagged1 expression activates Notch signaling in head and neck squamous cell carcinoma and promotes endothelial capillary-like sprout formation (24). HGF was found to induce hairy and enhancer of split-1 (HES-1) mRNA activation, resulting in the activation of Notch $(21,26)$. Moreover, the activation of c-Met was previously shown to stimulate Notch function in Drosophila (15). We found that Jagged1/Notch1 signaling could be triggered by HGF/c-Met signaling. Taken together, these findings suggest that, through MAPK and Hes-1 signal transduction, Jagged1/ Notch1 signaling functions downstream of c-Met.

The identification of patients with specific genetic mutations or amplifications has been applied in clinical target therapy for lung and breast cancer, and gastrointestinal stromal tumor. The Cancer Genome Atlas (TCGA) project divided

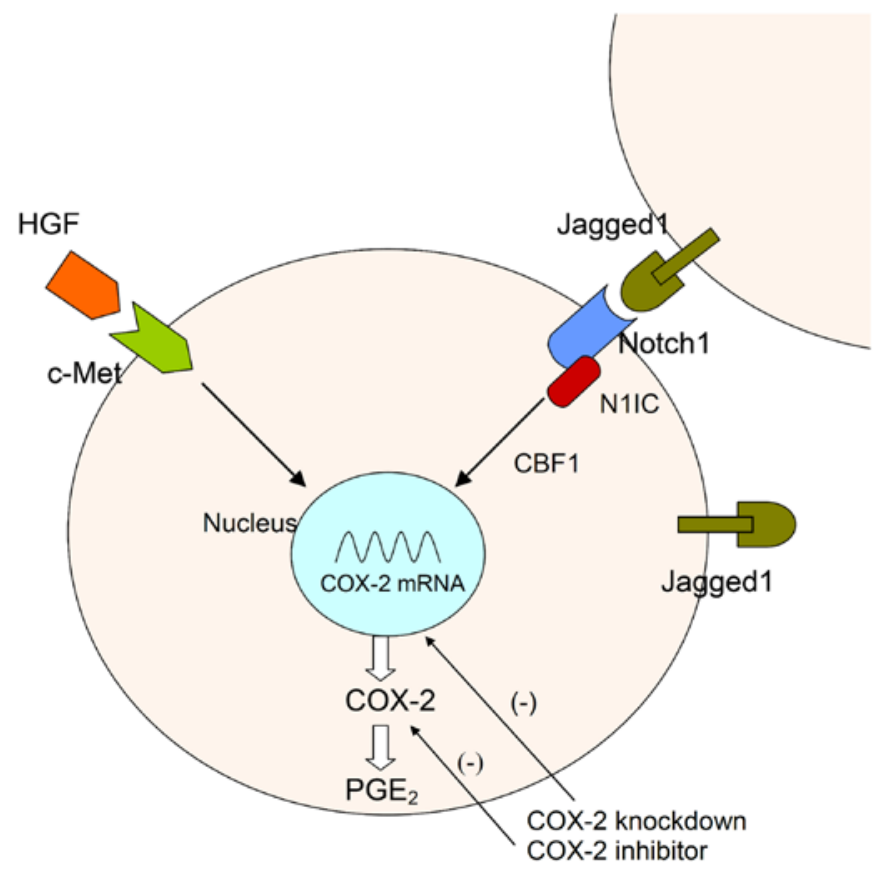

Figure 6. Signaling pathway between HGF/c-Met and Notch1/Jagged1. HGF binds to the c-Met receptor and activates the c-Met signaling pathway. The N1IC domain is cleaved and translocates into the nucleus after Jagged1mediated activation of Notch1 receptor. Both HGF/c-Met and Jagged1/ Notch1 pathways activate the COX-2 promoter and enhance cell proliferation and migration in human gastric cancer cells. HGF, hepatocyte growth factor; N1IC, Notch1 intracellular domain; $\mathrm{PGE}_{2}$, prostaglandin $\mathrm{E}_{2}$.

gastric cancer into four molecular subtypes: Epstein-Barr virus (EBV)-positive, microsatellite instability (MSI), genomically stable (GS), and chomosomal instability (CIN) (27). Targeted 
therapy toward human epidermal growth factor receptor 2 (Her-2 receptor) is applied to specific advanced gastric cancer patients with positive expression of Her-2/Neu (28). Recent studies have described carcinogenesis and the development of targeted therapy for c-Met signaling in gastric cancer $(6,29)$. Nickoloff $e t$ al also reported the biopharmacological potential of the Notch receptor as a targeted therapy for cancer (30). Notch ligand Jagged1 is also a potential pharmacogenomic target for cancer therapy (31). Inhibitory antibodies for c-Met and Notch receptors or inhibitors for Notch ligand Jagged1 may provide a therapeutic strategy for cancer on the basis of this signaling pathway. However, the interaction between HGF/c-Met and Jagged1/Notch1 signaling pathways should be considered. Both HGF/Notch1 and Jagged1/Notch1 signaling pathways target COX-2 mRNA, resulting in the production of COX-2 protein and the secretion of prostaglandin $\mathrm{E}_{2}\left(\mathrm{PGE}_{2}\right)$, and the elevation of the serum HGF level could induce the expression of Jagged1. Therefore, it is insufficient for the treatment of gastric cancer to involve the use of a single agent for a unique target receptor. Multi-modality targeted therapies should be considered for the therapeutic strategy for gastric cancer.

In conclusion, Jagged 1 expression is positively correlated with c-Met expression in human gastric cancer specimens. Constitutive activation of Notch1 limits HGF activity by repressing the c-Met oncogene. $\mathrm{HGF} / \mathrm{c}-\mathrm{Met}$ plays a minor role in the constitutive Notch1 signaling pathway. HGF/c-Met signaling induced an increase in Jagged1 expression in the control group in a time-dependent manner. Therefore, the reciprocal loop regulation between the HGF/c-Met and Jagged $1 /$ Notch signaling pathways plays an important role in modulating a biological response in gastric cancer cells (Fig. 6).

\section{Acknowledgements}

We thank Dr Shin-Shian Hsu for the suggestions regarding our manuscript.

\section{Funding}

The present study was supported by the Division of Experimental Surgery of the Department of Surgery in Taipei Veterans General Hospital, grants from Taipei Veterans General Hospital (V101B-035, V107C-043) and grants from the Ministry of Science and Technology (NSC 101-2314-B-075-074, MOST 104-2314-B-075-032-, 105-2314-B-075-005-MY2). No sources of funding played a role in the study design, data collection, analysis and interpretation of data, writing of the manuscript, and in the decision to submit the manuscript for publication.

\section{Availability of data and materials}

The datasets used during the present study are available from the corresponding author upon reasonable request.

\section{Authors' contributions}

CWC, MHY and YMS conceived and designed the study. CWW and WLF collected surgical specimens. KHH, ICS and AFYL performed the experiments. KHH wrote the study.
MHY, CWC, TSY, HCL and PHY reviewed and edited the manuscript. All authors read and approved the manuscript and agree to be accountable for all aspects of the research in ensuring that the accuracy or integrity of any part of the work are appropriately investigated and resolved.

\section{Ethics approval and consent to participate}

This study was approved by the Institutional Review Board of Taipei Veterans General Hospital and informed consent was provided by all subjects.

\section{Consent for publication}

Not applicable.

\section{Competing interests}

The authors declare that they have no competing interests.

\section{References}

1. Ferlay J, Soerjomataram I, Dikshit R, Eser S, Mathers C, Rebelo M, Parkin DM, Forman D and Bray F: Cancer incidence and mortality worldwide: Sources, methods and major patterns in GLOBOCAN 2012. Int J Cancer 136: E359-E386, 2015

2. Chen JH, Wu CW, Kao HL, Chang HM, Li AFY, Liu TY and Chi CW: Effects of COX-2 inhibitor on growth of human gastric cancer cells and its relation to hepatocyte growth factor. Cancer Lett 239: 263-270, 2006.

3. Yeh TS, Wu CW, Hsu KW, Liao WJ, Yang MC, Li AFY, Wang AM, Kuo ML and Chi CW: The activated Notch1 signal pathway is associated with gastric cancer progression through cyclooxygenase-2. Cancer Res 69: 5039-5048, 2009.

4. Jones MK, Sasaki E, Halter F, Pai R, Nakamura T, Arakawa T, Kuroki $\mathrm{T}$ and Tarnawski AS: HGF triggers activation of the COX-2 gene in rat gastric epithelial cells: Action mediated through the ERK2 signaling pathway. FASEB J 13: 2186-2194, 1999.

5. Amemiya H, Kono K, Itakura J, Tang RF, Takahashi A, An FQ, Kamei S, Iizuka $\mathrm{H}$, Fujii $\mathrm{H}$ and Matsumoto Y: c-Met expression in gastric cancer with liver metastasis. Oncology 63: 286-296, 2002.

6. Christensen JG, Burrows J and Salgia R: c-Met as a target for human cancer and characterization of inhibitors for therapeutic intervention. Cancer Lett 225: 1-26, 2005.

7. Chappell WH, Green TD, Spengeman JD, McCubrey JA, Akula SM and Bertrand FE: Increased protein expression of the PTEN tumor suppressor in the presence of constitutively active Notch-1. Cell Cycle 4: 1389-1395, 2005.

8. Lindsell CE, Shawber CJ, Boulter J and Weinmaster G: Jagged: A mammalian ligand that activates Notch1. Cell 80: 909-917, 1995.

9. Hsu KW, Hsieh RH, Huang KH, Fen-Yau Li A, Chi CW, Wang TY, Tseng MJ, Wu KJ and Yeh TS: Activation of the Notch1/STAT3/Twist signaling axis promotes gastric cancer progression. Carcinogenesis 33: 1459-1467, 2012.

10. Ohno R, Yoshinaga K, Fujita T, Hasegawa K, Iseki H, Tsunozaki H, Ichikawa W, Nihei Z and Sugihara K: Depth of invasion parallels increased cyclooxygenase-2 levels in patients with gastric carcinoma. Cancer 91: 1876-1881, 2001.

11. Shi H, Xu JM, Hu NZ and Xie HJ: Prognostic significance of expression of cyclooxygenase-2 and vascular endothelial growth factor in human gastric carcinoma. World J Gastroenterol 9: 1421-1426, 2003.

12. Lim HY, Joo HJ, Choi JH, Yi JW, Yang MS, Cho DY, Kim HS, Nam DK, Lee KB and Kim HC: Increased expression of cyclooxygenase-2 protein in human gastric carcinoma. Clin Cancer Res 6: 519-525, 2000.

13. Huang TT, Ping YH, Wang AM, Ke CC, Fang WL, Huang KH, Lee HC, Chi CW and Yeh TS: The reciprocal regulation loop of Notch2 pathway and miR-23b in controlling gastric carcinogenesis. Oncotarget 6: 18012-18026, 2015. 
14. Hsu KW, Fang WL, Huang KH, Huang TT, Lee HC, Hsieh RH, Chi CW and Yeh TS: Notch1 pathway-mediated microRNA$151-5 \mathrm{p}$ promotes gastric cancer progression. Oncotarget 7 : 38036-38051, 2016.

15. Stella MC, Trusolino L, Pennacchietti S and Comoglio PM: Negative feedback regulation of Met-dependent invasive growth by Notch. Mol Cell Biol 25: 3982-3996, 2005.

16. Wu CW, Chi CW, Su TL, Liu TY, Lui WY and P'eng FK: Serum hepatocyte growth factor level associate with gastric cancer progression. Anticancer Res 18: 3657-3659, 1998.

17. Wu CW, Li AFY, Chi CW, Chung WW, Liu TY, Lui WY and P'eng FK: Hepatocyte growth factor and Met/HGF receptors in patients with gastric adenocarcinoma. Oncol Rep 5: 817-822, 1998.

18. Lin JS, Lu CW, Huang CJ, Wu PF, Robinson D, Kung HJ, Chi CW, Wu CW, Yang WK, Whang-Peng JJ, et al: Proteintyrosine kinase and protein-serine/threonine kinase expression in human gastric cancer cell lines. J Biomed Sci 5: 101-110, 1998.

19. Uen YH, Lin SR, Wu CH, Hsieh JS, Lu CY, Yu FJ, Huang TJ and Wang JY: Clinical significance of MUC1 and c-Met RT-PCR detection of circulating tumor cells in patients with gastric carcinoma. Clin Chim Acta 367: 55-61, 2006.

20. Boccaccio C and Comoglio PM: Invasive growth: A MET-driven genetic programme for cancer and stem cells. Nat Rev Cancer 6 : 637-645, 2006.

21. Yamamoto $\mathrm{H}$, Itoh $\mathrm{F}$, Fukushima $\mathrm{H}$, Hinoda $\mathrm{Y}$ and Imai $\mathrm{K}$ : Overexpression of cyclooxygenase-2 protein is less frequent in gastric cancers with microsatellite instability. Int J Cancer 84: 400-403, 1999

22. Gual P, Giordano S, Williams TA, Rocchi S, Van Obberghen E and Comoglio PM: Sustained recruitment of phospholipase C- $\gamma$ to Gab1 is required for HGF-induced branching tubulogenesis. Oncogene 19: 1509-1518, 2000.

23. Maroun CR, Naujokas MA, Holgado-Madruga M, Wong AJ and Park M: The tyrosine phosphatase SHP-2 is required for sustained activation of extracellular signal-regulated kinase and epithelial morphogenesis downstream from the met receptor tyrosine kinase. Mol Cell Biol 20: 8513-8525, 2000.
24. Zeng Q, Li S, Chepeha DB, Giordano TJ, Li J, Zhang H, Polverini PJ, Nor J, Kitajewski J and Wang CY: Crosstalk between tumor and endothelial cells promotes tumor angiogenesis by MAPK activation of Notch signaling. Cancer Cell 8: 13-23, 2005.

25. King AM, Van der Put E, Blomberg BB and Riley RL: Accelerated Notch-dependent degradation of E47 proteins in aged B cell precursors is associated with increased ERK MAPK activation. J Immunol 178: 3521-3529, 2007.

26. Gude NA, Emmanuel G, Wu W, Cottage CT, Fischer K, Quijada P, Muraski JA, Alvarez R, Rubio M, Schaefer E, et al: Activation of Notch-mediated protective signaling in the myocardium. Circ Res 102: 1025-1035, 2008.

27. The Cancer Genome Atlas Research Network; Bass AJ, Thorsson V, Shmulevich I, Reynolds SM, Miller M, Bernard B, Hinoue T, Laird PW, Curtis C, Shen H, et al: Comprehensive molecular characterization of gastric adenocarcinoma. Nature 513: 202-209, 2014.

28. Gravalos C and Jimeno A: HER2 in gastric cancer: A new prognostic factor and a novel therapeutic target. Ann Oncol 19: $1523-1529,2008$

29. Peruzzi B and Bottaro DP: Targeting the c-Met signaling pathway in cancer. Clin Cancer Res 12: 3657-3660, 2006.

30. Nickoloff BJ, Osborne BA and Miele L: Notch signaling as a therapeutic target in cancer: A new approach to the development of cell fate modifying agents. Oncogene 22: 6598-6608, 2003.

31. Katoh M and Katoh M: Notch ligand, JAG1, is evolutionarily conserved target of canonical WNT signaling pathway in progenitor cells. Int J Mol Med 17: 681-685, 2006.

This work is licensed under a Creative Commons Attribution-NonCommercial-NoDerivatives 4.0 International (CC BY-NC-ND 4.0) License. 